\title{
Quantitative discrimination of Aggregatibacter actinomycetemcomitans highly leukotoxic JP2 clone from non-JP2 clones in diagnosis of aggressive periodontitis
}

Akihiro Yoshida ${ }^{*}$, Oum-Keltoum Ennibi ${ }^{2}$, Hideo Miyazaki ${ }^{3}$, Tomonori Hoshino $^{4}$, Hideaki Hayashida ${ }^{5}$, Tatsuji Nishihara ${ }^{6}$, Shuji Awano ${ }^{1}$ and Toshihiro Ansai ${ }^{1}$

\begin{abstract}
Background: Aggregatibacter actinomycetemcomitans is the etiological agent of periodontitis, and there is a strong association between clone JP2 and aggressive periodontitis in adolescents of African descent. The JP2 clone has an approximately 530-bp deletion $(\Delta 530)$ in the promoter region of the $\mathrm{kt} t / \mathrm{ltx}$ gene, which encodes leukotoxin, and this clone has high leukotoxic activity. Therefore, this clone is very important in aggressive periodontitis. To diagnose this disease, culture methods and conventional PCR techniques are used. However, quantitative detection based on qPCR for the JP2 clone has not been developed due to genetic difficulties. In this study, we developed a qPCR-based quantification method specific to the JP2 clone.

Methods: Based on our analysis of the DNA sequence of the $/ k t / l t x$ gene and its flanking region, we designed a reverse primer specific for the $\Delta 530$ deletion border sequence and developed a JP2-specific PCR-based quantification method using this primer. We also analyzed the DNA sequence of the $\triangle 530$ locus and found it to be highly conserved (97-100\%) among 17 non-JP2 strains. Using the $\triangle 530$ locus, we designed a qPCR primer-probe set specific to non-JP2 clones. Next, we determined the numbers of JP2 and non-JP2 clone cells in the periodontal pockets of patients with aggressive periodontitis.

Results: The JP2-specific primers specifically amplified the genomic DNA of the A. actinomycetemcomitans JP2 clone and did not react with other bacterial DNA, whereas the non-JP2 specific primers reacted only with $A$. actinomycetemcomitans non-JP2 clones. Samples from the 88 periodontal sites in the 11 patients with aggressive periodontitis were analyzed. The bacterial cell numbers in 88 periodontal sites ranged from 0 to $4.8 \times 10^{8}$ (mean $1.28 \times 10^{7}$ ) for JP2 clones and from 0 to $1.6 \times 10^{6}$ for non-JP2 clones (mean $1.84 \times 10^{5}$ ). There were significant differences in the JP2 cell number between a clinical attachment level (CAL) $\leq 6 \mathrm{~mm}$ and a level $\geq 7 \mathrm{~mm}(p<0.01)$. Our new QPCR-based JP2- and non-JP2-specific quantitative detection assay is applicable to the diagnosis of aggressive periodontitis with $A$. actinomycetemcomitans.
\end{abstract}

Conclusions: We successfully developed a quantitative and discriminative PCR-based method for the detection of A. actinomycetemcomitans JP2 and non-JP2 clones. This technique will contribute to future analyses of the quantitative relationship between this organism and aggressive periodontitis.

Keywords: Aggregatibacter actinomycetemcomitans, Aggressive periodontitis, JP2, Non-JP2, qPCR, Quantification

\footnotetext{
* Correspondence: akihiro@kyu-dent.ac.jp

'Division of Community Oral Health Science, Kyushu Dental College,

Kitakyushu, Japan

Full list of author information is available at the end of the article
} 


\section{Background}

Periodontitis is an infectious disease comprising a complex group of inflammatory conditions that eventually result in the loss of the teeth-supporting tissues [1]. Several systemic diseases are thought to be associated with periodontal disease [2-4]. Of the various forms of periodontitis, aggressive periodontitis is characterized by an early age of onset, with molar and incisor teeth the first ones affected in localized forms [5-7]. Studies have revealed an association between this disease and the presence of Aggregatibacter actinomycetemcomitans, a Gram-negative, facultative anaerobic coccobacillus [8-10]. A. actinomycetemcomitans isolated from aggressive periodontitis in adolescents of African descent living in different parts of the world are genetically homogeneous and belong to a single clone called JP2 [11-15]. The high pathogenicity of this clone has been supported by various epidemiological studies of people of African descent $[8,16]$. The pathogenicity of this clone is characterized by increased leukotoxin production. The highly leukotoxic JP2 clone of A. actinomycetemcomitans serotype b is characterized by a 530-bp deletion $(\Delta 530)$ in the promoter region of the $l k t / l t x$ gene operon, which encodes the leukotoxin, resulting in increased leukotoxin production [17-20]. This clone shows pronounced racial tropism, as it has been isolated almost exclusively from adolescent periodontitis patients of West and Northwest African descent [14,18]. Haubek et al. reported that the JP2 clone is an important etiological agent in the initiation of periodontal attachment loss in adolescents $[8,21,22]$.

Consequently, the accurate diagnosis of this disease is based on detecting the JP2 clone from periodontal lesions in patients with aggressive periodontitis. Previously, subgingival plaque cultures were used to analyze the association between the presence of the JP2 clone and aggressive periodontitis [23]. Since this is time consuming, detection using cultures is not suitable for rapid diagnosis. Molecular genetics techniques, which are relatively rapid, including conventional PCR and other molecular-based techniques, have been used to detect the JP2 clone from subgingival plaque [24,25]. However, these are qualitative methods, and no completely quantitative molecular-based detection technique for the JP2 clone has been reported [26]. Furthermore, a quantitative analysis of the A. actinomycetemcomitans JP2 clone and the severity of aggressive periodontitis has not been done, although positive relationships between the numbers of Porphyromonas gingivalis and Treponema denticola cells and the severity of adult periodontitis have been reported [27-29].

In this study, we developed a quantitative, discriminative detection method specific to A. actinomycetemcomitans JP2 and non-JP2 clones using qPCR (real-time PCR). This is the first report of quantitative detection specific to the A. actinomycetemcomitans JP2 and nonJP2 clones. Additionally, the relationship between the number of cells and the severity of this disease was examined. This method should contribute to clarifying the quantitative relationship between the JP2 clone and aggressive periodontitis.

\section{Results}

\section{Analysis of the A. actinomycetemcomitans IktC locus for} primer design

Figure 1 shows the gene cluster and nucleotide sequence of the region upstream from the $l t x C / l k t C$ locus. Downstream from the $g l y A$ gene, IGR1851 (IGR: non-coding region of genomic DNA, Gene ID: AA02808) was identified. The nucleotide sequence of IGR1851 of HK1651 is on the strand opposite that of Y4 and JP2 (Figure 1A). In the Y4 strain, the region upstream from $l t x C / l k t C$ contains a 762bp open-reading frame (ORF). The ORF of the JP2 clone, named $\operatorname{orf} X^{\prime}$, contains an approximately 530 -bp deletion compared with $\operatorname{orf} X$ of the non-JP2 strain. The 31-bp of sequence at the 3 ' end following the deletion is common to both $\operatorname{orf} X$ and $\operatorname{orf} X X^{\prime}$ (Figure 1B, double underlined). Therefore, we designed an antisense primer specific to the JP2 clone at the border of the $3^{\prime}$ region of orf $X^{\prime}$ and this 31-bp 3' locus of $\operatorname{orf} X$ (Figure 1B).

\section{Sequencing analysis of the $\Delta 530$ region for designing non-JP2 specific primers}

To evaluate the specificity of the $\Delta 530$ locus in the primer design, we analyzed the PCR products using primer pairs designed outside the $\Delta 530$ locus. Initially, we performed PCR analysis using the primer pair Aa orfX3'F1 and Aa lktC5'R1 (Figure 1A, Table 1). Of the 18 non-JP2 strains, seven were positive using this primer set (723 bp, Table 2). To amplify the $\Delta 530$ locus of the other strains, primer pair Aa orfX3'F2 and Aa lktC5'R2 was also designed (Figure 1A, Table 1). Using these primers, in 10 of 11 nonJP2 strains, except for NCTC 9710, the locus 3' of orf $X$ was amplified (809 bp, Table 2). The DNA sequences of the $\Delta 530$ locus of 17 strains were analyzed using these amplicons. Using the DNA sequence data, homology analysis was performed among these 17 strains (Additional file 1: Table S2). Of the 17 non-JP2 strains, the $\Delta 530$ region consisted of 528 bp for strains Y4 and ATCC 29522 and $531 \mathrm{bp}$ for the other strains (Additional file 1: Table S2). The homologies were between 96.987 and 100\%. Therefore, the $\Delta 530$ locus was highly conserved among non-JP2 strains. Furthermore, the homology search revealed that the nucleotide sequences of the $\Delta 530$ locus were specific to the non-JP2 strains (blastn, data not shown). Therefore, we considered the $\Delta 530$ locus to be a nucleotide marker for non-JP2 strains and designed a qPCR primer set and hydrolysis probe specific to the nonJP2 strains in this region (Table 1). 


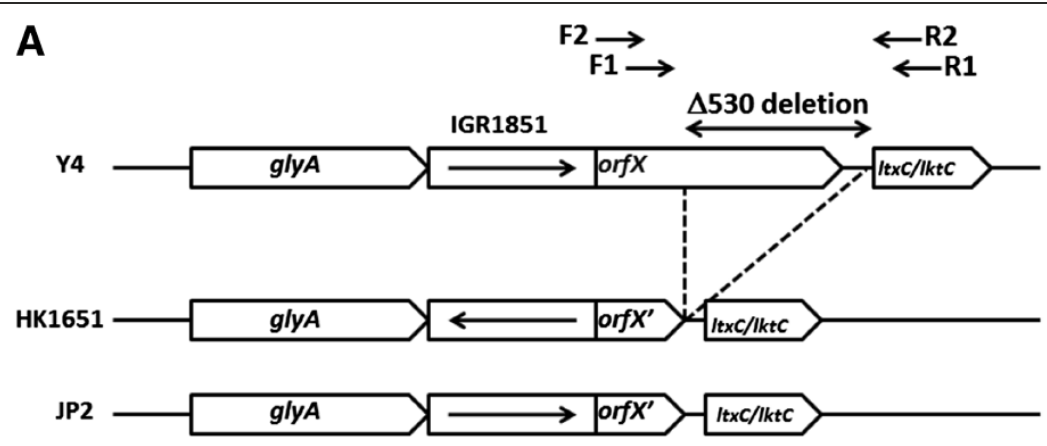

B

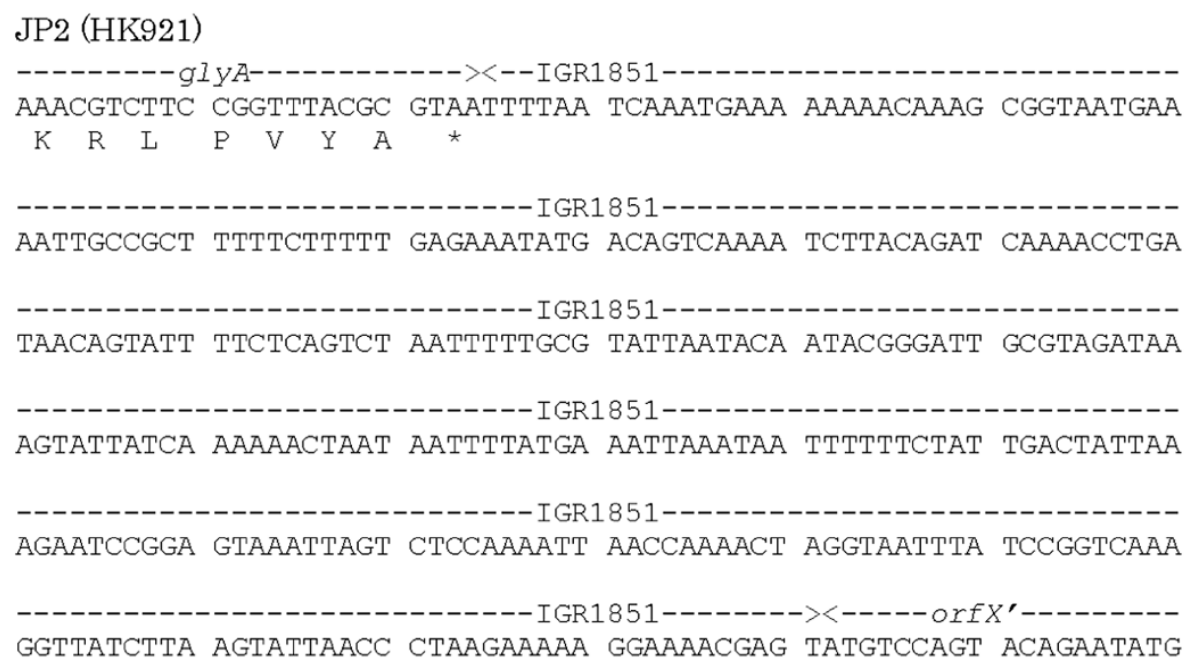

------------------------ OrfX'--------------Forward primer

CTCCATTTTA TCTCCGTTTT ATTCAGTTCC CAAGTAATGA AGTTTTACTC TATGAATACT

GGAAACTTGT TCAGATTTT GTACAAAAGg TTAGTAAAAT AACGGTAAGA TTAGCACAAA

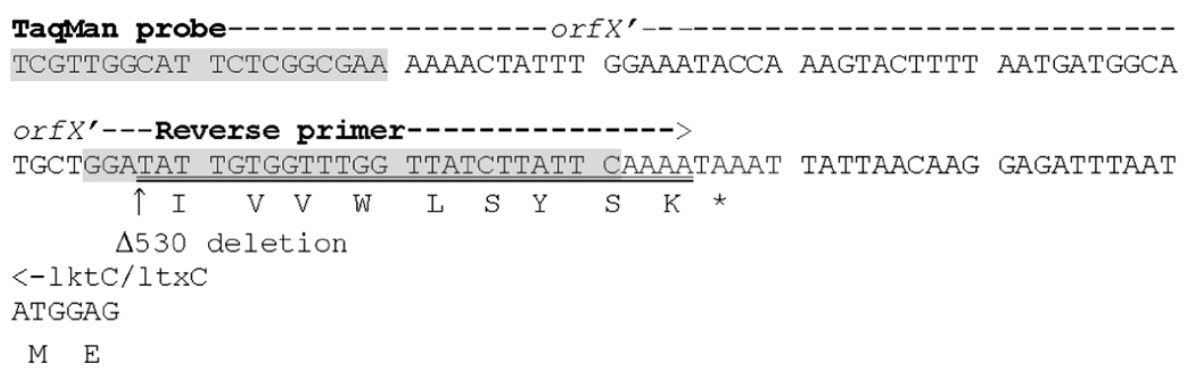

Figure 1 Comparison of (A) the genetic organization of JP2 clones and non-JP2 strains and (B) the nucleotide sequences of the glyA gene and orfX (orfX)-IktC locus of the JP2 strain. (A) The $\triangle 530$-bp deletion of the JP2 strain was identified. F1 and R1, Aa orfX3'F1 and Aa IktC5'R1; F2 and R2, Aa orfX3'F2 and Aa lktC5'R2. (B) The nucleotide sequence of the JP2-specific primer-probe set. The arrow indicates the $\Delta 530$-bp deletion. The double underlined amino acid sequence (IMVLSYSK) overlaps the $3^{\prime}$ locus of orfX.

Specificity and sensitivity of the JP2- and non-JP2-specific primers

Using these primer pairs and probe, we confirmed the primers' specificity by checking their reactivity with various strains of $A$. actinomycetemcomitans and other oral bacteria (Tables 2 and Additional file 2: Table S1).
The JP2-specific primers specifically amplified the genomic DNA of the A. actinomycetemcomitans JP2 clone and did not react with other bacterial DNA, whereas the non-JP2 specific primers reacted only with $A$. actinomycetemcomitans non-JP2 clones (Tables 2 and Additional file 2: Table S1). The sensitivity of this 
Table 1 Oligonucleotide primers and hydrolysis probes used in this study

\begin{tabular}{|c|c|c|c|}
\hline Primers or Probes & Sequence & Gene & Size \\
\hline \multicolumn{4}{|l|}{ Primer } \\
\hline$J P 2-F 3$ & 5'-TCT ATG AAT ACT GGA AAC TTG TTC AGA AT-3' & orf $x^{\prime}$ & $151 \mathrm{bp}^{\mathrm{d}}$ \\
\hline$J P 2-R 2$ & 5'-GAA TAA GAT AAC CAA ACC ACA ATA TCC-3' & orfx' & \\
\hline Non-JP2F & 5'-CGC AAG TGC CAT AGT TAT CCA CT-3' & orfx ( $\triangle 530$ region) & $145 b p^{e}$ \\
\hline Non-JP2R & 5'-TCG TCT GCG TAA TAA GCA AGA GAG-3' & orfX ( $\triangle 530$ region) & \\
\hline Aa orfX3'F1 & 5'-AAA TCG TTG GCA TTC TCG -3' & orf $x$ & $723 b p^{e}$ \\
\hline Aa lktC5'R1 & 5'-CAA AGG AGA ATT TGC CCA -3' & IktC & \\
\hline Aa orfX3'F2 & 5'-CCG TTT TAT TCA GTT CCC -3' & orf $x$ & $809 \mathrm{bp}^{\mathrm{e}}$ \\
\hline Aa lktC5'R2 & 5'-TGC CCA TAA CCA AGC CAC -3' & IktC & \\
\hline \multicolumn{4}{|l|}{ Hydrolysis probes ${ }^{a}, b, c$} \\
\hline JP2 & 5'-FAM-ACA AAT CGT TGG CAT TCT CGG CGA A-TAMRA-3' & orfx' & \\
\hline Non-JP2 & 5'-FAM-ATA TTG TAG ACA TCG CCC-MGB-3' & orfx ( $\triangle 530$ region) & \\
\hline
\end{tabular}

${ }^{\mathrm{a} F A M,}$ 6-carboxyfluorescein.

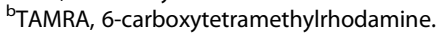

${ }^{\mathrm{C}} \mathrm{MGB}$, minor groove binder.

${ }^{d}$ calculated in JP2 strain.

${ }^{\mathrm{e}}$ calculated in Y4 strain.

TaqMan assay for A. actinomycetemcomitans JP2 and non-JP2 clones was evaluated. Serially diluted chromosomal DNA of A. actinomycetemcomitans JP2 and non-JP2 clones was used to evaluate the lower detection limits. The lower detection limit was $2.19 \mathrm{pg} /$ mixture for the JP2 clone and $2.24 \mathrm{pg} /$ mixture for the non-JP2 clone. The standard curves for linear regressions between the threshold cycle $(\mathrm{Ct})$ values and corresponding colony forming units (CFU) were obtained. The standard curves for $A$. actinomycetemcomitans JP2 and non-JP2 were $Y=-3.548 X+40.96, \mathrm{R}^{2}=1.00$ and $Y=-3.501 X+36.91, \mathrm{R}^{2}=1.00(Y=\mathrm{Ct}, X=\mathrm{CFU})$, respectively (data not shown). The detection limits of the assays determined with serial dilutions of $A$. actinomycetemcomitans JP2 and Y4 genomic DNA were 4 to $4 \times 10^{6}$ copies (for JP2, corresponding to 4 to $4 \times$ $10^{6} \mathrm{CFU}$ ) and 11 to $1.1 \times 10^{7}$ copies (for Y4, corresponding to 11 to $1.1 \times 10^{7} \mathrm{CFU}$ ), respectively (data not shown).

\section{Inhibitory effect of oral specimens on the assay (spike analysis)}

The presence of inhibitors in the oral specimens was assessed using lysates spiked with dental plaque (ca. $1 \mathrm{~g}$ [wet weight] per mixture, to mimic subgingival plaque) without the $A$. actinomycetemcomitans JP2 clone. The presence of dental plaque caused negligible inhibition of the real-time PCR amplification (data not shown). A similar spike experiment was performed using the non-JP2 specific primers and dental plaque without $A$. actinomycetemcomitans strains. Dental plaque had no inhibitory effects on the assay (data not shown).
Quantification of the JP2 and non-JP2 clones from subgingival plaque samples

Samples from the 88 periodontal sites (31 incisors, 44 first molars, 13 premolars) in the 11 patients with aggressive periodontitis were analyzed. Nine of the 11 patients were positive for JP2 clones, and three of these nine were also positive for non-JP2 clones. Two patients were negative for both clones. Forty-six sites were positive for JP2 clones (52.3\%); of these, nine sites were positive for both JP2 and non-JP2 clones (10.2\%). One site was positive only for non-JP2 clones (1.1\%). Neither clone was detected at 39 sites (44.3\%). The bacterial cell numbers in 88 periodontal sites ranged from 0 to $4.8 \times$ $10^{8}$ (mean $1.28 \times 10^{7}$ ) for JP2 clones and from 0 to $1.6 \times$ $10^{6}$ for non-JP2 clones (mean $1.84 \times 10^{5}$ ).

\section{Correlation between periodontal status and the number of JP2 cells}

All samples were divided into two groups based on the CAL, and the mean number of JP2 clone cells was determined. There was a significant difference in the number between groups with CAL $\leq 6 \mathrm{~mm}$ (mean $4.74 \mathrm{~mm}$ ) and that with CAL $\geq 7 \mathrm{~mm}$ (mean $9.12 \mathrm{~mm})(p<0.01$, Table 3). The analysis of non-JP2 was not clear due to the small number of non-JP2 positive samples $(n=10$, data not shown).

\section{Discussion}

Studies have shown that highly leukotoxic A. actinomycetemcomitans strains, i.e., the JP2 clone, are associated with aggressive periodontitis [8]. A cross-sectional study of Moroccan adolescents found a strong association 
Table 2 Primer specificity for the Aggregatibacter actinomycetemcomitans strains

\begin{tabular}{|c|c|c|c|c|c|c|}
\hline \multirow[t]{2}{*}{ Strain } & \multirow[t]{2}{*}{ Serotype } & \multirow[t]{2}{*}{ JP2/non-JP2 } & \multicolumn{2}{|c|}{ Amplification with primer pair: $^{a}$} & \multirow{2}{*}{$\begin{array}{l}\text { Primer pairs } \\
\text { for } \Delta 530 \text { locus } \\
\text { analysis }^{\text {b }} \text { (for non-JP2) }\end{array}$} & \multirow[t]{2}{*}{ Source or reference } \\
\hline & & & JP2F3-JP2R2 & NonJP2F- NonJP2R & & \\
\hline ATCC 29523 & $a$ & non-JP2 & - & + & 1 & ATCC $^{C}$ \\
\hline SUNYaB 75 (ATCC 43717) & a & non-JP2 & - & + & 1 & SUNYaB ${ }^{d}$ \\
\hline $\mathrm{TN}-1$ & $\mathrm{a}$ & non-JP2 & - & + & 2 & Nishihara $^{e}$ \\
\hline Y4 (ATCC 43718) & $b$ & non-JP2 & - & + & 1 & Socransky ${ }^{f}$ \\
\hline JP2 (HK921) & $b$ & $\mathrm{JP} 2$ & + & - & - & Kilian ${ }^{g}$ \\
\hline HK 1199 & $b$ & $\mathrm{JP} 2$ & + & - & - & Kilian \\
\hline HK 1519 & $b$ & $J P 2$ & + & - & - & Kilian \\
\hline HK 1611 & $\mathrm{~b}$ & $J P 2$ & + & - & - & Kilian \\
\hline HK 1612 & $\mathrm{~b}$ & $J P 2$ & + & - & - & Kilian \\
\hline HK 1651 & $\mathrm{~b}$ & $\mathrm{JP} 2$ & + & - & - & Kilian \\
\hline HK 1709 & $b$ & non-JP2 & - & + & 1 & Kilian \\
\hline ATCC 29522 & $b$ & non-JP2 & - & + & 2 & ATCC \\
\hline ATCC 29524 & $\mathrm{~b}$ & non-JP2 & - & + & 1 & ATCC \\
\hline HK 916 & c & non-JP2 & - & + & 1 & Kilian \\
\hline SUNYaB 67 (ATCC 43719) & c & non-JP2 & - & + & 1 & SUNYaB \\
\hline NCTC 9709 & c & non-JP2 & - & + & 2 & NCTC \\
\hline NCTC 9710 & c & non-JP2 & - & + & none & NCTC \\
\hline 3381 & $d$ & non-JP2 & - & + & 2 & Asikainen ${ }^{i}$ \\
\hline IDH 781 & $d$ & non-JP2 & - & + & 2 & Asikainen \\
\hline OMZ 534 & e & non-JP2 & - & + & 2 & Gmürj \\
\hline OMZ 541 & e & non-JP2 & - & + & 2 & Gmür \\
\hline OMZ 546 & e & non-JP2 & - & + & 2 & Gmür \\
\hline NUM 5005 & $f$ & non-JP2 & - & + & 2 & Takadak \\
\hline NUM 4039 & g & non-JP2 & - & + & 2 & Takada \\
\hline
\end{tabular}

a + , positive amplification with a band of the expected size; -, no amplification observed.

b1, Aa orfX3'F1 and Aa IktC5'R1; 2, Aa orfX3'F2 and Aa lktC5'R2 (Table 1); none, not 1 or 2s.

${ }^{c}$ ATCC, American Type Culture Collection, Rockville, Md.

${ }^{\mathrm{d}}$ SUNYaB, State University of New York at Buffalo, NY.

${ }^{\mathrm{e}}$ Nishihara, T. Nishihara, Kyushu Dental College, Kitakyushu, Japan.

fSocransky, S. Socransky, Forsyth Dental Center, Mass.

${ }^{9}$ Kilian, M. Kilian, University of Aarhus, Aarhus, Denmark.

${ }^{\mathrm{h}} \mathrm{NCTC}$, National Collection of Type Cultures, London, England.

'Asikainen, S. Asikainen, University of Helsinki, Helsinki, Finland.

${ }^{\mathrm{j}}$ Gmür, R. Gmür, University of Zurich, Zurich, Switzerland.

kTakada, K. Takada, Nihon University School of Dentisry at Matsudo, Chiba, Japan.

between the presence of the JP2 clone and periodontal attachment loss [23]. Furthermore, a population-based longitudinal study found an increased risk of developing periodontal attachment loss when the JP2 clone of $A$. actinomycetemcomitans was present in the subgingival plaque $[8,30]$. However, these investigations were based

Table 3 The number of JP2 clones in 88 subgingival plaque samples and the grouping in terms of the CAL

\begin{tabular}{lcc}
\hline CAL & Mean \pm SD of bacterial cell number & $P^{a}$ \\
\hline$\leq 6 \mathrm{~mm}(\mathrm{n}=31)$ & $3.79 \times 10^{5} \pm 1.58 \times 10^{5}$ & 0.003 \\
$\geq 7 \mathrm{~mm}(\mathrm{n}=57)$ & $1.92 \times 10^{7} \pm 8.35 \times 10^{7}$ & \\
\hline
\end{tabular}

${ }^{a}$ Mann-Whitney test. on qualitative evaluations of the JP2 strain, i.e., the relationship between the presence or absence of JP2 and the severity of aggressive periodontitis. The quantitative relationship between the number of JP2 cells and the severity of aggressive periodontitis had not been discussed prior to this report. A previous investigation reported a differentiation and quantification method for A. actinomycetemcomitans JP2 and 652 strains [26]. However, this differentiation system was based on amplicon size differences and requires agarose gel electrophoresis or melting-curve analysis to distinguish these strains. Therefore, the assay is not a primer-specificity-based method. Consequently, we developed both JP2-clone- 
and non-JP2-clone-specific quantification methods based on primer specificity and the qPCR technique.

First, we analyzed the nucleotide sequences of the promoter region of the $l t x C / l k t C$ gene. For this purpose, we analyzed the PCR products using the primer pairs Aa orfX3'F1 and Aa lktC5'R1 or Aa orfX3'F2 and Aa lktC5'R2. However, the orfX-ltxC/lktC locus was not amplified only in the NCTC 9710 strain using these primers. In addition, using the primer pair Aa orf3'F1-Aa lktC5'R2 and Aa orf3'F2-Aa lktC5'R1, the orfX-ltxC/lktC locus in the NCTC 9710 strain was not amplified by PCR. We postulated that the DNA sequences of these primers differ from those of the NCTC 9710 strain, but this is no more than a supposition. As described previously, the most dramatic variation is an approximately 530-bp deletion in the JP2 leukotoxin promoter relative to the non-JP2 strain $[17,18,20]$. The 530-bp deletion removes a DNA segment starting in the middle of orf $X$, a 762-bp ORF found in Y4 and ATCC 33384, and continues to a position 25 bp 5 ' to the $l+x C / l k t C$ start codon. Additionally, IGR1851 was identified between the $g l y A$ and $l t x C / l k t C$ genes. According to the Oralgen database (http://www.oralgen.lanl.gov/_index.html) of the Los Alamos National Laboratory, the nucleotide sequence of IGR1851 of HK1651 is on the strand opposite that of Y4 and JP2 (Figure 1A). Consequently, we designed a sense primer and hydrolysis probe inside orf $X^{\prime}$ and the antisense primer border of the $3^{\prime}$ region of $\operatorname{orf} X^{\prime}$ and $3^{\prime}$ of orf $X$ (Figure 1B, double underlined). For the JP2 clone, the sense and antisense JP2-specific primers anneal to the appropriate loci on $\operatorname{orf} X^{\prime}$ (Figure 2). For the non-JP2 clone, however, the sense primer anneals to $\operatorname{orf} X^{\prime}$, while the antisense primer is not able to anneal to the $3^{\prime}$ border of $\operatorname{orf} X^{\prime}$ due to the lack of 530-bp deletion (Figure 2). This primer design successfully amplified only the JP2 clone. Conversely, non-JP2 specific oligonucleotide primers were designed inside the $\Delta 530$-bp locus. To analyze the conservation and specificity of the $\Delta 530$-bp locus, we sequenced it, revealing that the $\Delta 530$-bp locus is highly conserved among non-JP2 strains (ranging from 96.987 to $100 \%)$. The non-JP2-specific primer sets successfully amplified non-JP2 strains, but not the JP2 strain. As shown in Table 2, the specificities of these assays were evaluated. Additionally, we examined the effect of nontarget DNA on this assay, and the influence of the copresence of non-target DNA was negligible (data not shown). This spiking experiment showed that this characteristic is suited to detection in oral specimens. Next, we evaluated the sensitivities of this assay. Using serially diluted chromosomal DNA (see results), we found that the sensitivities of these assays were consistent with previous studies [27,31]. Using this assay, we detected and quantified both JP2 and non-JP2 clones from subgingival plaque samples from adolescents.

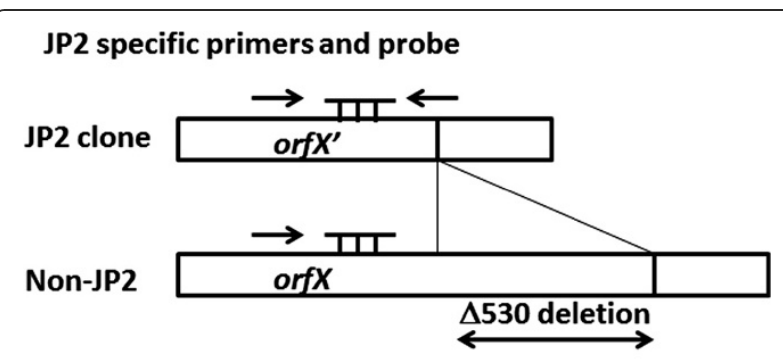

Non-JP2 specific primers and probe

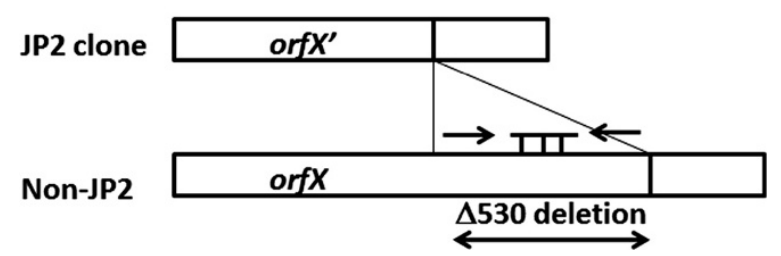

Figure 2 Designation of the oligonucleotide primers and probes specific to the JP2 clone (above) and non-JP2 strains (below). For the JP2-specific amplification, the sense primer and hydrolysis probe are complementary to orfx' (for JP2 clone) and orfX (for non-JP2 strains). The antisense primer was designed to be complementary to the border of the $3^{\prime}$ end of orf $X^{\prime}$ for the specific amplification of JP2 clones. For non-JP2-specific selection, the sense and antisense primers and TaqMan probe were designed to be complementary to the orf $\triangle 530$ region.

In this study, $48(54.5 \%)$ of the 88 periodontal sites (in nine of 11 individuals) were positive for JP2 clones. Previous studies reported that the prevalence of the JP2 strain in Moroccan adolescents was 50 of $428(11.7 \%)$ individuals [8] and 43 of 365 (11.8\%) individuals [30]. Our study subjects were aggressive periodontitis patients, whereas the other studies included subjects with healthy periodontium. Therefore, those data cannot strictly be compared with our results. Additionally, the JP2 and non-JP2 clones were detected using conventional PCR in those studies. Seki et al. applied the loop-mediated isothermal amplification (LAMP) method for detecting the JP2 clone in subgingival plaque from Moroccan adolescents and found that 48 of 72 samples (66.7\%) were JP2 positive [25]. The LAMP technology was very sensitive for the JP2 clone, and the detection limit was 10 genome copies, whereas that with conventional PCR was 100 copies $[25,32,33]$. These findings are identical to the sensitivity of $\mathrm{qPCR}$, and the detection rate was similar to our result $(54.5 \%)$. In this study, we quantified the number of cells of the JP2 and non-JP2 clones. We also analyzed the relationship between cell number and CAL. There was a significant difference in JP2 cell number between group with a CAL $\leq 6 \mathrm{~mm}$ and that with $\geq 7 \mathrm{~mm}(p<0.01)$. The mean JP2 cell number of the CAL $>7 \mathrm{~mm}$ group was more than 50 times that of the CAL $<6 \mathrm{~mm}$ group. Previously, several population-based studies demonstrated that 
the presence of the A. actinomycetemcomitans JP2 clone increased the risk of progression of CAL over a 2-year period $[8,22]$. However, those were qualitative studies using conventional PCR. We showed that the number of JP2 cells was associated with the severity of aggressive periodontitis, making our study the first to reveal a quantitative relationship between the JP2 strain and CAL in aggressive periodontitis. This strongly supports the findings that the $A$. actinomycetemcomitans JP2 clone is an agent in the etiology of aggressive periodontitis. However, the quantitative analysis of aggressive periodontitis has just started, and further molecular-based epidemiological studies are required.

Finally, combined with population-based studies, our investigation will contribute to elucidating the quantitative relationship between the number of $A$. actinomycetemcomitans JP2 clones and the severity of aggressive periodontitis. These fundamental data will allow risk assessment to determine prevention and treatment strategies for this disease.

\section{Conclusions}

We present the first method for the quantitative detection of $A$. actinomycetemcomitans JP2 and non-JP2 clones. The technique is based on real-time PCR methodology with TaqMan probe and primers specifically designed to discriminate these clones. This method was used to quantify $A$. actinomycetemcomitans JP2 in the periodontal pockets of patients with aggressive periodontitis, and revealed a significant difference in the number of $A$. actinomycetemcomitans JP2 cells between subjects with CALs $\leq 6 \mathrm{~mm}$ and those with CALs $\geq 7$ $\mathrm{mm}(p<0.01)$. This technique will contribute to future investigations of the quantitative relationship between $A$. actinomycetemcomitans and aggressive periodontitis.

\section{Methods}

\section{Bacterial strains and culture conditions}

The A. actinomycetemcomitans strains listed in Table 2 were grown in Trypticase soy broth (Becton Dickinson, Sparks, MD) supplemented with $0.6 \%$ yeast extract (Becton Dickinson) and $0.04 \%$ sodium bicarbonate at $37^{\circ} \mathrm{C}$ in a $5 \%$ $\mathrm{CO}_{2}$ atmosphere [34]. Other oral bacteria used in this study are listed as supplemental data (Additional file 2: Table S1).

\section{DNA techniques}

Routine molecular biology techniques were performed as described by Sambrook et al. [35]. Chromosomal DNA was isolated from the bacteria listed in Tables 2 and Additional file 2: Table S1 using a Puregene DNA isolation kit (Gentra Systems, Minneapolis, MN). Nucleotide sequence information for A. actinomycetemcomitans HK1651 was obtained from the Oral Pathogen
Sequence Database (Los Alamos National Laboratory, http://www.oralgen.lanl.gov/).

\section{DNA sequencing of the $\Delta \mathbf{5 3 0}$ region of orfX}

The genetic structures of the JP2 clone and non-JP2 strain are shown in Figure 1A. To design highly specific oligonucleotide primers for real-time PCR-based detection of $A$. actinomycetemcomitans non-JP2 strains, the $\triangle 530$ region of orf $X$ was sequenced using two oligonucleotide primer pairs (Table 1). Initially, Aa orfX3'F1 (137-154 bp from the 5' end of orf $X$ ) and Aa lktC5'R1 (49-66 bp from the 5' end of $l k t C$ ) were used for all strains, and Aa orfX3'F2 (33-50 bp from the 5' end of $\operatorname{orf} X)$ and Aa lktC5'R2 (37-54 bp from the 5' end of $l k t C$ ) were used for Aa orfX3'F1- and Aa lktC5'R1negative strains (Table 2). The PCR conditions consisted of $95^{\circ} \mathrm{C}$ for $5 \mathrm{~min}$, followed by 25 cycles of $95^{\circ} \mathrm{C}$ for $30 \mathrm{~s}$, $47^{\circ} \mathrm{C}$ for $30 \mathrm{~s}$, and $72^{\circ} \mathrm{C}$ for $1 \mathrm{~min}$ for primers $\mathrm{Aa}$ orfX3'F1 and Aa lktC5'R1 and $95^{\circ} \mathrm{C}$ for $5 \mathrm{~min}$, followed by 25 cycles of $95^{\circ} \mathrm{C}$ for $30 \mathrm{~s}, 49^{\circ} \mathrm{C}$ for $30 \mathrm{~s}$, and $72^{\circ} \mathrm{C}$ for 1 min for primers Aa orfX3'F2 and Aa lktC5'R2. DNA sequencing of these PCR amplicons, performed using an Applied Biosystems 3730 DNA Analyzer (Applied Biosystems, Foster City, CA) and BigDye Terminator v3.1 Cycle Sequence Kit (Applied Biosystems) at Hokkaido System Science (Hokkaido, Japan), yielded a consensus sequence and allowed us to design non-JP2 strainspecific primers (Table 1).

\section{Primers and probe design}

A hydrolysis probe-based qPCR assay (TaqMan assay) was used for the specific detection of $A$. actinomycetemcomitans JP2 and non-JP2 strains. The oligonucleotide primers and hydrolysis probe were designed from the orf $X^{\prime}$ region and its 3' border sequences (for JP2 clones; Oral Pathogen Sequence Database number: AA02808) and $\Delta 530$ region of orf $X$ (for non-JP2 strains) using Primer Express 3.0 (Applied Biosystems). As shown in Table 1, the hydrolysis probe specific for the JP2 clone was labeled with 6-carboxyfluorescein (FAM) and 6-carboxytetramethylrhodamine (TAMRA) and for the non-JP2 clone the hydrolysis probe was labeled with FAM and minor groove binder (MGB). The primer specificity was initially confirmed by gapped blastn 2.0.5 (National Center for Biotechnology Information, http://www.ncbi.nlm.nih.gov/) analysis and then by conventional PCR performed using chromosomal DNA from various oral bacteria (Table 2 and Additional file 2: Table S1). These PCR assays were performed using thermocycler conditions of $94^{\circ} \mathrm{C}$ for 5 min, followed by 25 cycles of $94^{\circ} \mathrm{C}$ for $30 \mathrm{~s}, 60^{\circ} \mathrm{C}$ for $30 \mathrm{~s}$, and $72^{\circ} \mathrm{C}$ for $1 \mathrm{~min}$ for the JP2 clone and $94^{\circ} \mathrm{C}$ for $5 \mathrm{~min}$, followed by 25 cycles of $94^{\circ} \mathrm{C}$ for $30 \mathrm{~s}, 49^{\circ} \mathrm{C}$ for $30 \mathrm{~s}$, and $72^{\circ} \mathrm{C}$ for $1 \mathrm{~min}$ for non-JP2 clones. 


\section{qPCR}

Chromosomal DNA from bacterial cultures and clinical specimens and a negative control sample were amplified using LightCycler TaqMan Master (Roche Diagnostics $\mathrm{GmbH}$, Mannheim, Germany). The amplification reaction mixture consisted of DNA template, $5 \times$ Master Mix, forward and reverse primers $(0.5 \mu \mathrm{M}$ each), $0.2 \mu \mathrm{M}$ hydrolysis probe, and PCR-grade water in a LightCycler Capillary (Roche Diagnostics). Specific products were amplified and detected using the LightCycler Carouselbased System (Roche Diagnostics), with the following thermal conditions: $95^{\circ} \mathrm{C}$ for $10 \mathrm{~min}$, followed by 45 cycles of $95^{\circ} \mathrm{C}$ for $10 \mathrm{~s}, 58^{\circ} \mathrm{C}$ for $40 \mathrm{~s}$, and $72^{\circ} \mathrm{C}$ for $1 \mathrm{~s}$ for both JP2 and non-JP2 strains. The endpoint amplification products were further separated on $2 \%$ agarose gels to confirm that they were of the predicted sizes.

\section{Study subjects}

Eleven adolescents and young adults 16-31 years old (three males, eight females; mean \pm SD age $26.6 \pm 4.1$ years), diagnosed with aggressive periodontitis at the Dental Hospital, Université Mohammed V Souissi in Rabat, Morocco, participated in this study. Subjects were excluded if they used antibiotics within the preceding year, were undergoing ongoing periodontal treatment (scaling, root planing, and professional tooth cleaning), had orthodontic or prosthetic devices, had used mouth rinse routinely in the previous 6 months, or were smokers.

\section{Ethics statement}

All patients were treated in accordance with the Declaration of Helsinki on the participation of human subjects in medical research. Ethics clearance for the study was obtained from Comité d'Ethique pour la Recherché Biomédicale (CERB), Université Mohammed V Souissi (reference number 400). Patients were fully informed and signed informed consent forms.

\section{Clinical examination}

The clinical attachment level (CAL) in the periodontium was measured as the distance in millimeters from the cement-enamel junction to the bottom of the periodontal pocket using a periodontal probe at eight sites (i.e., the buccal aspects of the mesial surfaces of the upper and lower first incisors and the first molars; if a tooth was missing, the premolar on the same side was used), for a total of 88 sites. To determine the reproducibility of the CAL recordings, all subjects were examined twice at a 2-week interval using identical procedures. The same examiner (O.-K.E.) performed both examinations, but was not aware of the initial findings at the time of the second examination. The reproducibility of recording the CAL was evaluated by measuring the kappa value, which was 0.65 .

\section{Microbiological sampling and preparation}

Subgingival samples on paper points were obtained from the adolescents, as described previously [8]. Briefly, subgingival plaque samples were obtained by inserting a sterile paper point into a subgingival site for $10 \mathrm{~s}$. The paper point was transferred into $200 \mu$ l of phosphatebuffered saline $(\mathrm{PBS})$ and centrifuged at $12,000 \times g$ for 5 min. After centrifugation, the bacteria chromosomal DNA was extracted using MORA-extract (Cosmo Bio, Tokyo, Japan), according to the manufacturer's instructions, and then served as a template. After centrifugation, the supernatant was removed, and $150 \mu \mathrm{l}$ of lysis buffer were added to the pellet. The lysed bacteria were transferred to a tube with glass beads and heated at $90^{\circ} \mathrm{C}$ for $10 \mathrm{~min}$. Then, the bacterial mixture was disrupted using a Mini-Bead Beater (Biospec Products, Bartlesville, OK) with 0.1-mm-diameter glass beads at 4,800 rpm for $2 \mathrm{~min}$. After this procedure, $200 \mu \mathrm{l}$ of SDS solution were added and heated at $90^{\circ} \mathrm{C}$ for $10 \mathrm{~min}$. Then, $400 \mu \mathrm{l}$ of phenol solution were added and mixed for $1 \mathrm{~min}$. After centrifugation, the aliquot was subjected to ethanol precipitation and dissolved in $20 \mu \mathrm{l}$ of TE buffer.

\section{Statistical analysis}

Intergroup bacterial cell numbers were analyzed using the Mann-Whitney test. The kappa value was measured to determine the reproducibility of recording the CAL. Statistical analysis was performed with the program SPSS 11.0 (SPSS, Chicago, IL), and the differences were considered significant when the $p$-value was less than 0.05 .

\section{Additional files}

Additional file 1: Table S2. Homology analysis of the $\triangle 530$ region of A. actinomycetemcomitans non-JP2 strains.

Additional file 2: Table S1. Bacterial strains other than Aggregatibacter actinomycetemcomitans used for primer-specificity analysis.

\section{Abbreviations}

qPCR: Quantitative polymerase chain reaction; CAL: Clinical attachment level; CFU: Colony forming unit; Ct: Threshold cycle; FAM: 6-carboxy-fluoresein; TAMRA: 6-carboxy-tetramethylrhodamine; MGB: Minor groove binder.

\section{Competing interests}

The authors declare that they have no competing interests.

\section{Authors' contributions}

AY participated in the design of the study, perfected the DNA manipulations, performed qPCR analyses, wrote the first draft of the manuscript, and was responsible for the overall coordination of the study. O-KE performed the clinical examinations and samplings of subgingival plaque at a dental hospital in Rabat, Morocco. HM provided the contacts with the staff of the Faculté de Médecine Dentaire, Université Mohammed V Souissi, Rabat, Morocco. TH and HH organized and performed the microbiological studies. TN organized and supervised the microbiological studies. TA and SA conducted the statistical analyses. All authors collaboratively interpreted the results, were involved in writing the manuscript, and approved the final version. 


\section{Acknowledgments}

The authors thank Prof. Mogens Kilian, Department of Biomedicine, Aarhus University, Denmark, for helpful suggestions. Support for this study was provided by Grants-in-Aid (C) 22592341 (A.Y.), (C) 21592644 (H.M.), and (B) 22390403 (T.A.) from the Ministry of Education, Culture, Sports, Science and Technology of Japan.

\section{Author details}

${ }^{1}$ Division of Community Oral Health Science, Kyushu Dental College, Kitakyushu, Japan. ²Département de Parodontologie, Faculté de Médecine Dentaire, Université Mohammed V Souissi, Rabat, Morocco. ${ }^{3}$ Department of Oral Health Science, Niigata University Graduate School of Medical and Dental Science, Niigata, Japan. ${ }^{4}$ Department of Pediatric Dentistry, Nagasaki University Graduate School of Biomedical Sciences, Nagasaki, Japan. ${ }^{5}$ Department of Preventive Dentistry, Nagasaki University Graduate School of Biomedical Sciences, Nagasaki, Japan. ${ }^{6}$ Division of Infections and Molecular Biology, Kyushu Dental College, Kitakyushu, Japan.

Received: 17 July 2012 Accepted: 9 October 2012

Published: 11 October 2012

\section{References}

1. van Dyke $T E$, Serhan $C N$ : Resolution of inflammation: a new paradigm for the pathogenesis of periodontal diseases. J Dent Res 2003, 82:82-90.

2. Friedewald VE, Kornman KS, Beck JD, Genco R, Goldfine A, Libby P, Offenbacher S, Ridker PM, Van Dyke TE, Roberts WC: American Journal of Cardiology; Journal of Periodontology: The American Journal of Cardiology and Journal of Periodontology Editors' Consensus: periodontitis and atherosclerotic cardiovascular disease. Am J Cardiol 2009, 104:59-68

3. Ishihara K, Nabuchi A, Ito R, Miyachi K, Kuramitsu HK, Okuda K: Correlation between detection rates of periodontopathic bacterial DNA in coronary stenotic artery plaque and in dental plaque samples. J Clin Microbiol 2004, 42:1313-1315. Erratum in: J Clin Microbiol 2004, 42:5437.

4. Offenbacher $\mathrm{S}$, Boggess KA, Murtha AP, Jared $\mathrm{HL}$, Lieff $\mathrm{S}$, McKaig RG, Mauriello SM, Moss KL, Beck JD: Progressive periodontal disease and risk of very preterm delivery. Obstet Gynecol 2006, 107:29-36.

5. American Academy of Periodontology: Parameter on aggressive periodontitis. J Periodontol 2000, 71:867-869.

6. Pihlstrom BL, Fine $\mathrm{DH}$ : Aggressive periodontitis in adolescents in Morocco. Lancet 2008, 371:188-189.

7. Rylev M, Bek-Thomsen M, Reinholdt J, Ennibi OK, Kilian M: Microbiological and immunological characteristics of young Moroccan patients with aggressive periodontitis with and without detectable Aggregatibacter actinomycetemcomitans JP2 infection. Mol Oral Microbiol 2011, 26:35-51.

8. Haubek D, Ennibi OK, Poulsen K, Vaeth M, Poulsen S, Kilian M: Risk of aggressive periodontitis in adolescent carriers of the JP2 clone of Aggregatibacter (Actinobacillus) actinomycetemcomitans in Morocco: a prospective longitudinal cohort study. Lancet 2008, 371:237-242.

9. Nørskov-Lauritsen N, Nørskov-Lauritsen M: Reclassification of Actinobacillus actinomycetemcomitans, Haemophilus aphrophilus, Haemophilus paraphrophilus and Haemophilus segnis as Aggregatibacter actinomycetemcomitans gen. nov., comb. nov., Aggregatibacter aphrophilus comb. nov. and Aggregatibacter segnis comb. nov., and emended description of Aggregatibacter aphrophilus to include $V$ factordependent and V factor-independent isolates. Int J Syst Evol Microbiol 2006, 56:2135-2146.

10. Zambon JJ: Actinobacillus actinomycetemcomitans in human periodontal disease. J Clin Periodontol 1985, 12:1-20.

11. Haubek DM, Dirienzo J, Tinoco EM, Westergaard J, Lopez NJ, Chung CP, Poulsen K, Kilian M: Racial tropism of a highly toxic clone of Actinobacillus actinomycetemcomitans associated with juvenile periodontitis. J Clin Microbiol 1997, 35:3037-3042.

12. Haubek D, Poulsen K, Asikainen S, Kilian M: Evidence for absence in northern Europe of especially virulent clonal types of Actinobacillus actinomycetemcomitans. J Clin Microbiol 1995, 33:395-401.

13. Haubek D, Poulsen K, Kilian M: Microevolution and patterns of dissemination of the JP2 clone of Aggregatibacter (Actinobacillus) actinomycetemcomitans. Infect Immun 2007, 75:3080-3088.
14. Haubek D, Poulsen K, Westergaard J, Dahlèn G, Kilian M: Highly toxic clone of Actinobacillus actinomycetemcomitans in geographically widespread cases of juvenile periodontitis in adolescents of African origin. J Clin Microbiol 1996, 34:1576-1578.

15. Rylev M, Kilian M: Prevalence and distribution of principal periodontal pathogens worldwide. J Clin Periodontol 2008, 35:346-361.

16. Cortelli SC, Costa FO, Kawai T, Aquino DR, Franco GC, Ohara K, Roman-Torres CV, Cortelli JR: Diminished treatment response of periodontally diseased patients infected with the JP2 clone of Aggregatibacter (Actinobacillus) actinomycetemcomitans. J Clin Microbiol 2009, 47:2018-2025.

17. Brogan JM, Lally ET, Poulsen K, Kilian M, Demuth DR: Regulation of Actinobacillus actinomycetemcomitans leukotoxin expression: analysis of the promoter regions of leukotoxic and minimally leukotoxic strains. Infect Immun 1994, 62:501-508.

18. Contreras A, Rusitanonta T, Chen C, Wagner WG, Michalowicz BS, Slots J: Frequency of 530-bp deletion in Actinobacillus actinomycetemcomitans leukotoxin promoter region. Oral Microbiol Immunol 2000, 15:338-340.

19. He T, Nishihara T, Demuth DR, Ishikawa I: A novel insertion sequence increases the expression of leukotoxicity in Actinobacillus actinomycetemcomitans clinical isolates. J Periodontol 1999, 70:1261-1268.

20. Kolodrubetz D, Spitznagel J Jr, Wang B, Phillips LH, Jacobs C, Kraig E: cis Elements and trans factors are both important in strain-specific regulation of the leukotoxin gene in Actinobacillus actinomycetemcomitans. Infect Immun 1996, 64:3451-3460.

21. Haubek D, Ennibi OK, Abdellaoui L, Benzarti N, Poulsen S: Attachment loss in Moroccan early onset periodontitis patients and infection with the JP2-type of Actinobacillus actinomycetemcomitans. J Clin Periodontol 2002, 29:657-660.

22. Haubek D, Ennibi OK, Poulsen K, Benzarti N, Baelum V: The highly leukotoxic JP2 clone of Actinobacillus actinomycetemcomitans and progression of periodontal attachment loss. J Dent Res 2004, 83:767-770.

23. Haubek D, Ennibi OK, Poulsen K, Poulsen S, Benzarti N, Kilian M: Early-onset periodontitis in Morocco is associated with the highly leukotoxic clone of Actinobacillus actinomycetemcomitans. J Dent Res 2001, 80:1580-1583.

24. Poulsen K, Ennibi OK, Haubek D: Improved PCR for detection of the highly leukotoxic JP2 clone of Actinobacillus actinomycetemcomitans in subgingival plaque samples. J Clin Microbiol 2003, 41:4829-4832.

25. Seki M, Poulsen K, Haubek D, Kilian M: Novel loop-mediated isothermal amplification method for detection of the JP2 clone of Aggregatibacter actinomycetemcomitans in subgingival plaque. J Clin Microbiol 2008, 46:1113-1115.

26. Orrù G, Marini MF, Ciusa ML, Isola D, Cotti M, Baldoni M, Piras V, Pisano E, Montaldo C: Usefulness of real time PCR for the differentiation and quantification of 652 and JP2 Actinobacillus actinomycetemcomitans genotypes in dental plaque and saliva. BMC Infect Dis 2006, 6:98.

27. Kawada M, Yoshida A, Suzuki N, Nakano Y, Saito T, Oho T, Koga T: Prevalence of Porphyromonas gingivalis in relation to periodontal status assessed by real-time PCR. Oral Microbiol Immunol 2004, 19:289-292.

28. Simonson LG, Goodman CH, Bial JJ, Morton HE: Quantitative relationship of Treponema denticola to severity of periodontal disease. Infect Immun 1988, 56:726-728.

29. Yoshida A, Kawada M, Suzuki N, Nakano Y, Oho T, Saito T, Yamashita Y: TaqMan real-time polymerase chain reaction assay for the correlation of Treponema denticola numbers with the severity of periodontal disease. Oral Microbiol Immunol 2004, 19:196-200.

30. Haubek D, Ennibi OK, Vaeth M, Poulsen S, Poulsen K: Stability of the JP2 clone of Aggregatibacter actinomycetemcomitans. J Dent Res 2009, 88:856-860

31. Yoshida A, Suzuki N, Nakano Y, Oho T, Kawada M, Koga T: Development of a 5' fluorogenic nuclease-based real-time PCR assay for quantitative detection of Actinobacillus actinomycetemcomitans and Porphyromonas gingivalis. J Clin Microbiol 2003, 41:863-866.

32. Leys EJ, Griffen AL, Strong SJ, Fuerst PA: Detection and strain identification of Actinobacillus actinomycetemcomitans by nested PCR. J Clin Microbiol 1994, 32:1288-1294.

33. Osawa R, Yoshida A, Masakiyo Y, Nagashima S, Ansai T, Watari H, Notomi T, Takehara T: Rapid detection of Actinobacillus actinomycetemcomitans 
using a loop-mediated isothermal amplification method. Oral Microbiol Immunol 2007, 22:252-259.

34. Sreenivasan PK, LeBlanc DJ, Lee LN, Fives-Taylor P: Transformation of Actinobacillus actinomycetemcomitans by electroporation, utilizing constructed shuttle plasmids. Infect Immun 1991, 59:4621-4627.

35. Sambrook J, Fritsch EF, Maniatis T: Molecular cloning: a laboratory manual. 2nd edition. Cold Spring Harbor, N.Y:: Cold Spring Harbor Laboratory Press; 1989 .

doi:10.1186/1471-2334-12-253

Cite this article as: Yoshida et al:: Quantitative discrimination of Aggregatibacter actinomycetemcomitans highly leukotoxic JP2 clone from non-JP2 clones in diagnosis of aggressive periodontitis. BMC Infectious Diseases 2012 12:253.

\section{Submit your next manuscript to BioMed Central and take full advantage of:}

- Convenient online submission

- Thorough peer review

- No space constraints or color figure charges

- Immediate publication on acceptance

- Inclusion in PubMed, CAS, Scopus and Google Scholar

- Research which is freely available for redistribution 